\title{
Closed-form object restoration from limited spatial and spectral information
}

\author{
Robert J. Marks II and Michael J. Smith \\ Department of Electrical Engineering FT-10, University of Washington, Seattle, Washington 98195
}

Received July 16, 1981

\begin{abstract}
Given only a portion of an arbitrary finite-energy $\left[L_{2}(-\infty, \infty)\right]$ image and a portion of that image's spectrum, we present a closed-form method by which the entire image can be reconstructed. The algorithm is a basic augmentation of a recently proposed iterative restoration algorithm presented by Stark et al. [J. Opt. Soc. Am. 71, 635 (1981); Opt. Lett. 6, 259 (1981)]. Experimental results are presented.
\end{abstract}

Building on the mathematical foundation laid by Youla, ${ }^{1}$ Stark et al. ${ }^{2,3}$ recently presented an iterative algorithm for reconstruction of an $L_{2}$ signal with only partial knowledge of the signal in the spatial and frequency domain. In this Letter we show that the algorithms can be straightforwardly placed in closed form when they are implemented digitally. This treatment of the algorithm of Stark et al. is directly parallel to Sabri and Steenaart's ${ }^{4}$ closed-form treatment of Gerchberg's iterative extrapolation algorithm..$^{5,6}$

\section{Development}

A closed-form restoration matrix will first be developed for example A of Ref. 2. Following the identical notation, we have, from Eq. (24) of Ref. 2,

$$
f_{k}(x)=\sum_{r=0}^{k-1}\left(Q_{b} \mathcal{P}_{a}\right)^{r} m_{1}(x)
$$

where $m_{1}$ is formed from the two known projections of $f(x)$ :

$$
m_{1}(x)=\mathscr{P}_{b} f(x)+\mathcal{Q}_{b}\left[Q_{a} f(x)\right] .
$$

When they are implemented discretely in one dimension, the projection operators take on the form of matrices. The matrix $P_{a}$ corresponding to the operator $\mathcal{P}_{a}$ is simply ${ }^{4}$

$$
P_{a}=\operatorname{diag}\{0,0, \ldots, 0,1, \ldots, 1,1,1, \ldots, 1,0, \ldots, 0,0\},
$$

i.e., a matrix everywhere zero except for 1's appropriately placed along the diagonal. The operator

$$
\mathscr{P}_{b}=1-Q_{b}
$$

is simply a low-pass filter matrix that can be formed by

$$
P_{b}=D^{-1} B D,
$$

where $D$ is the discrete Fourier-transform matrix and

$$
B=\operatorname{diag}\{0,0, \ldots, 0,1, \ldots, 1,1,1, \ldots, 1,0, \ldots, 0,0\}
$$

deletes all frequency components outside the interval $|\omega| \leq b$. Other formulations of the low-pass matrix are also possible. ${ }^{7}$ The $Q_{b}$ matrix is obtained by

$$
Q_{b}=I-P_{b},
$$

where $I$ is the identity matrix.

\section{Restoration Matrix}

Let $\mathbf{f}_{k}$ denote a vector of samples from $f_{k}(x)$. The restoration algorithm then becomes

$$
\begin{aligned}
\mathbf{f}_{k} & =\sum_{r=0}^{k-1}\left(Q_{b} P_{a}\right)^{r} \mathbf{m}_{1} \\
& =R_{k} \mathbf{m}_{1},
\end{aligned}
$$

where the $k$ th-order restoration matrix

$$
R_{k}=\sum_{r=0}^{k-1}\left(Q_{b} P_{a}\right)^{r}
$$

can be computed off line.

If we let $k$ tend to $\infty$, then, assuming convergence, ${ }^{4}$

$$
\begin{aligned}
R & \triangleq R_{\infty} \\
& =\sum_{r=0}^{\infty}\left(Q_{b} P_{a}\right)^{r} \\
& =\left(I-Q_{b} P_{a}\right)^{-1},
\end{aligned}
$$

where we have utilized a generalized geometric series. Then

$$
\mathbf{f}_{\infty}=R \mathbf{m}_{1} .
$$

The restoration matrix can thus be formed iteratively or, as here, through matrix inversion.

The above analysis can be generalized such that $f(x)$ and $F(\omega)$ are known over any interval. The choice of these intervals, however, dictates whether the problem is well or ill posed. ${ }^{1,2}$

\section{Experimental Results}

To illustrate application of Eq. (2), we now present restoration of a number of degraded signals. Each 
consists of 34 samples. The duration corresponding to $\mathcal{P}_{a}$ in each example is 8 of the 34 points. Filtering was performed with a Sabri-Steenaart low-pass filter ${ }^{7}$ with bandwidth $\beta=7$. In each of the six figures, points are connected for clarity of presentation.

The topmost function in Figs. 1-3 is the original image. Figure 1 is a single pulse, Fig. 2 is a single-sided

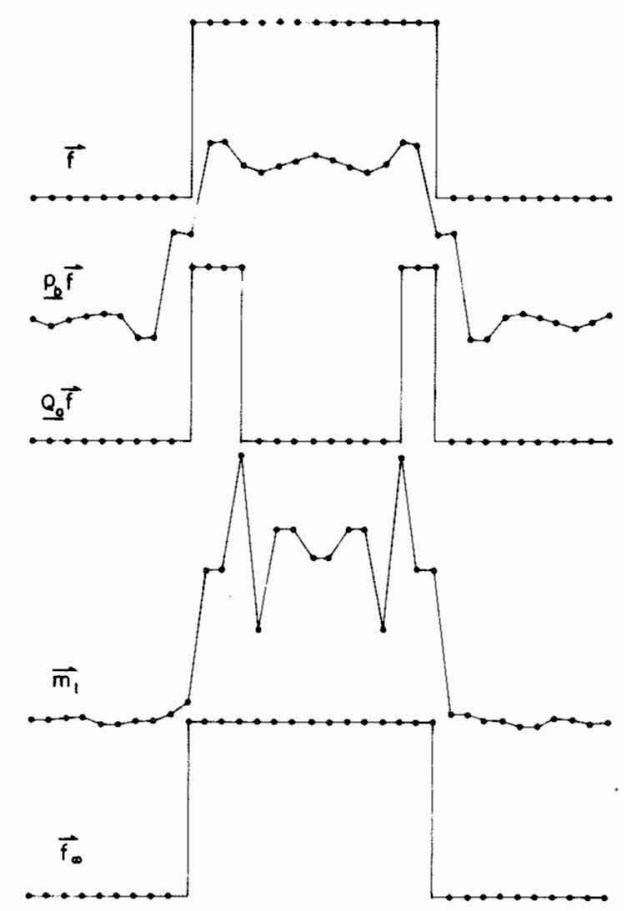

Fig. 1. Restoration of a pulse from $\grave{P}_{b} \mathbf{f}$ and $Q_{a} \mathbf{f}$.

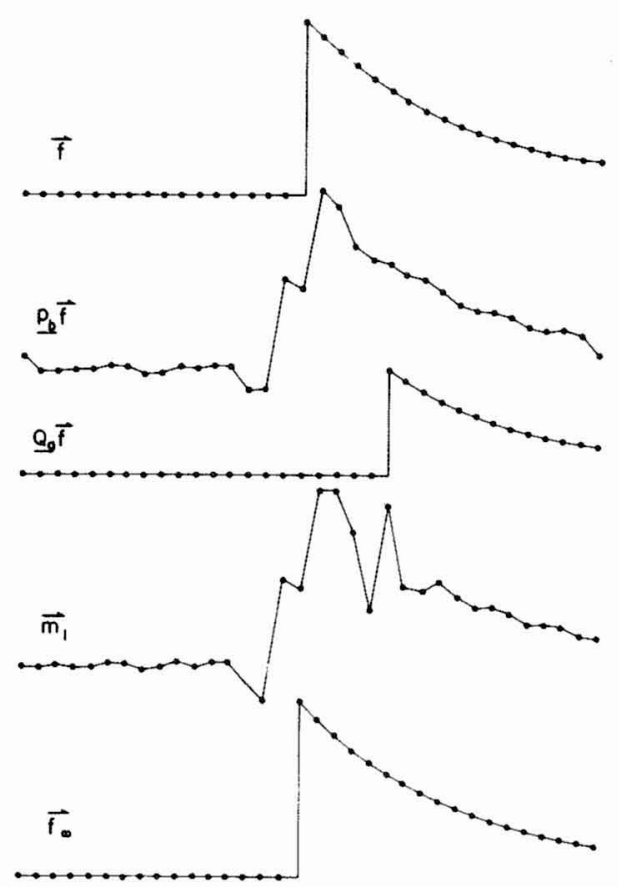

Fig. 2. Restoration of a single-sided exponential from $P_{b} \mathbf{f}$ and $Q_{a} \mathbf{f}$.

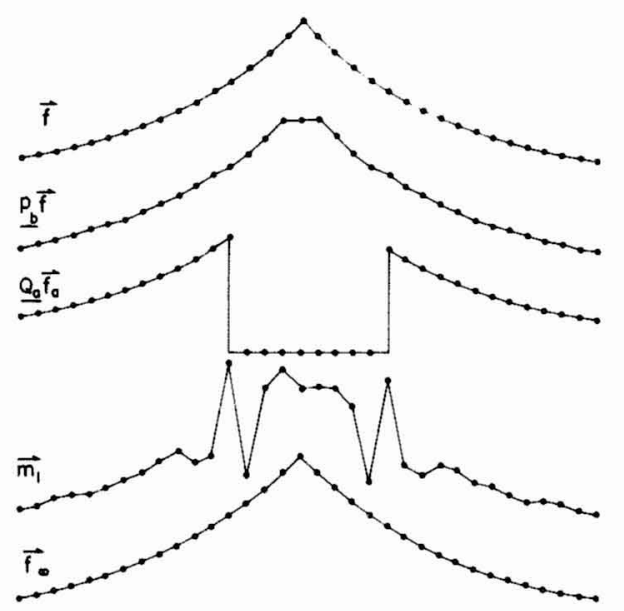

Fig. 3. Restoration of a double-sided exponential from $P_{b} \mathbf{f}$ and $Q_{a} \mathbf{f}$.

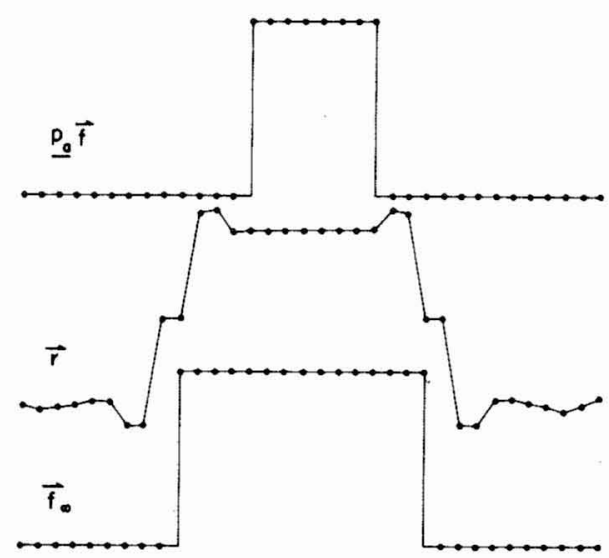

Fig. 4. Restoration of a pulse from $P_{b} f$ and $P_{a} \mathbf{f}$. The vectors $\mathbf{f}$ and $P_{b} \mathbf{f}$ are shown in Fig. 1.

exponential, and Fig. 3 is a double-sided exponential. Each figure also contains the assumed known portions of $\mathbf{f}: \mathbf{P}_{\mathrm{b}} \mathbf{f}$ and $Q_{a} \mathbf{f}$. The function $m_{1}$ is the linear combination of these projections corresponding to ${ }^{1}$

$$
\mathbf{m}_{1}=P_{b} \mathbf{f}+Q_{b} Q_{a} \mathbf{f} .
$$

The bottom function in Figs. 1-3 shows the restored image computed by Eq. (2). In each case, the restoration agreed with the image to six places.

A second set of examples was performed for the illposed problem presented in Ref. 3 reconstructing $f$ from $\mathcal{P}_{a} f$ and $\mathcal{P}_{b} f$. When an identical procedure is followed, as before, the restoration matrix here becomes

$$
R=\left(I-Q_{a} Q_{b}\right)^{-1} .
$$

We form a linear combination of the known data, as in Eq. (10) of Ref. 3:

$$
\mathbf{r}=P_{a} \mathbf{f}+Q_{a} P_{b} \mathbf{f}
$$

and obtain the restoration 


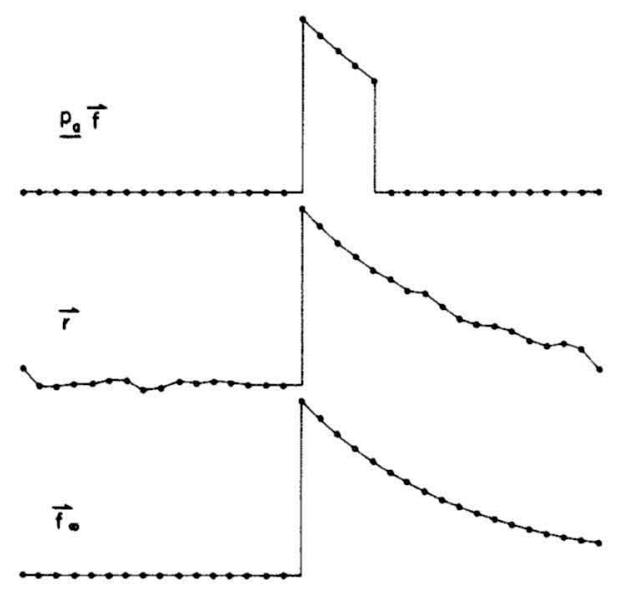

Fig. 5. Restoration of a single-sided exponential from $P_{b} \mathbf{f}$ and $P_{a}$ f. The vectors $\mathbf{f}$ and $P_{b} \mathbf{f}$ are shown in Fig. 2.

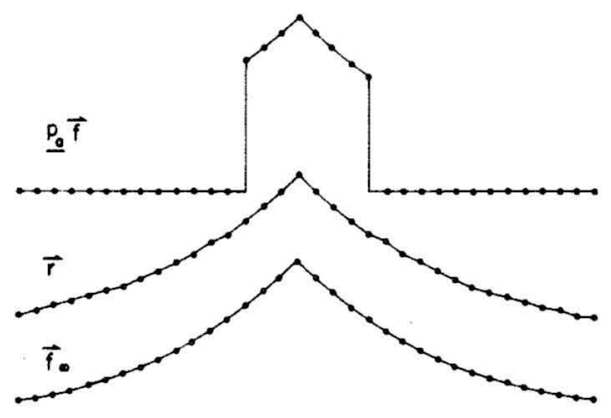

Fig. 6. Restoration of a double-sided exponential from $P_{b} \mathbf{f}$ and $P_{a} \mathbf{f}$. The vectors $f$ and $P_{b} \mathbf{f}$ are shown in Fig. 3.

$$
\mathbf{f}_{\infty}=R \mathbf{r} \text {. }
$$

Results for identical images are shown in Figs. 4-6. In each case, the restoration again agreed with the image to six places.
Continuous ill-posed problems manifest themselves digitally as ill-conditioned matrices. ${ }^{8}$ In the presence of even minute noise, the restoration results can vary wildly, as was illustrated in certain of the extrapolations of Smith and Marks. ${ }^{9}$

The authors gratefully acknowledge the support of this work by the National Science Foundation under grant ENG 7908009.

\section{References}

1. D. C. Youla, "Generalized image restoration by method of alternating projections," IEEE Trans. Circuits Syst. CAS-25, 694-702 (1978).

2. H. Stark, D. Cahana, and H. Webb, "Restoration of arbitrary finite-energy optical objects from limited spatial and spectral information," J. Opt. Soc. Am. 71, 635-642 (1981).

3. H. Stark, D. Cahana, and G. J. Habetler, "Is it possible to restore an optical object from its low-pass spectrum and its truncated image?" Opt. Lett. 6, 259-260 (1981).

4. M. S. Sabri and W. Steenaart, "An approach to bandlimited signal extrapolation: the extrapolation matrix," IEEE Trans. Circuits Syst. CAS-25, 74-78 (1978).

5. R. W. Gerchberg, "Super-resolution through error energy reduction," Opt. Acta 21, 709-720 (1974).

6. A. Papoulis, "A new algorithm in spectral analysis and band-limited extrapolation," IEEE Trans. Circuits Syst. CAS-22, 735-742 (1975).

7. M. S. Sabri and W. Steenaart, "Discrete Hilbert transform filtering," IEEE Trans. Acoust. Speech Signal Process. ASSP-25, 452-454 (1977).

8. T. K. Sarkar, D. D. Weiner, and V. K. Jain, "Some mathematical considerations in dealing with the inverse problem," IEEE Trans. Antennas Propag. AP-29, 373-379 (1981).

9. D. K. Smith and R. J. Marks II, "Closed form bandlimited image extrapolation,” Appl. Opt. 20, 2476-2483 (1981). 